\title{
KCNQ2 Encephalopathy Showing a Distinct Ictal Ampli- tude-Integrated Electroencephalographic Pattern
}

Naeun Kwak, MD, Yun Jeong Lee, MD, Dongsub Kim, MD, Su-Kyeong Hwang, MD, Soonhak Kwon, MD, and Eun Joo Lee, MD

Department of Pediatrics, School of Medicine, Kyungpook National University, Daegu, Korea

\section{ABSTRACT}

KCNQ2 mutations induce a neonatal-onset epileptic encephalopathy of widely varying severity, ranging from benign familial neonatal epilepsy to severe refractory epileptic encephalopathy. Refractory seizures with KCNQ2 mutations have a positive response to sodium-channel blockers. Recently, a distinctive ictal pattern has been reported during amplitude-integrated electroencephalographic (aEEG) monitoring in infants with $K C N Q 2$ encephalopathy. Herein, we describe a case of $K C N Q 2$ encephalopathy with this distinctive ictal aEEG pattern, which was confirmed using conventional electroencephalography (EEG). A 3-day-old female infant presented with neonatal seizures accompanied by cyanosis and desaturation. Her seizure semiology was tonic and focal clonic. Her ictal aEEG demonstrated a sudden rise in amplitude followed by a suppressed background pattern. This pattern was also confirmed on conventional EEG. Her seizures were refractory despite the administration of multiple conventional antiepileptic drugs. Finally, c.794C $>$ T; p. (Ala265Val) mutation was observed in the KCNQ2 gene on genetic testing, and she was diagnosed with KCNQ2 encephalopathy. Identifying this distinctive ictal pattern on aEEG monitoring facilitates the early detection of $K C N Q 2$ encephalopathy and timely targeted treatment in patients with refractory seizures.

Key Words: KCNQ2; Mutation; Infant; Seizures; Epilepsy; Electroencephalography

\section{INTRODUCTION}

Developmental and epileptic encephalopathies (DEE) are a group of progressive early onset epilepsies derived from genetic etiologies and characterized by developmental delay and cognitive deterioration ${ }^{1)}$. Genetic epilepsy should be considered as a differential diagnosis when a patient with neonatal seizures does not have a clear history of hypoxic ischemic encephalopathy, vascular events, or infection.

The development of gene sequencing techniques has led to an increasing identification of genetic causes for $\mathrm{DEE}^{2}$. Targeted gene panel testing revealed a diagnostic yield of $42.9 \%$ and
Received: 5 August 2020

Revised: 12 October 2020

Accepted: 15 October 2020

Correspondence to: Eun Joo Lee, MD

Department of Pediatrics, Kyungpook National University Hospital, School of Medicine, Kyungpook National University, 130 Dongdeok-ro, Jung-gu, Daegu 41944, Korea

Tel: +82-53-200-5704

Fax: +82-53-425-6683

E-mail:pshmom00@gmail.com

Copyright(c)

By Korean Society of Neonatology.

All right reserved.

This is an Open-Access article distributed under the terms of the Creative Commons Attribution Non-Commercial License (http:// creativecommons.org/licenses/by-nc/4.0), which permits unrestricted non-commercial use, distribution, and reproduction in any medium, provided the original work is properly cited. 
$65.2 \%$ in recent studies from South Korea and the United States, respectively ${ }^{1,3)}$. Mutations in $K C N Q 2$ are frequently identified in patients with $\mathrm{DEE}^{1,3)}$ are located on the chromosome (20q13.33) encoding the voltage-dependent potassium channel subunit 7.2 (Kv7.2), and manifest as a broad spectrum of neonatal-onset epilepsy with a varying severity from benign familial neonatal seizures to KCNQ2 encephalopathy ${ }^{4-6)}$.

$K C N Q 2$ encephalopathy is associated with refractory seizures resulting in a poor neurodevelopmental outcome. Many patients with DEE have seizures refractory to multiple antiepileptic drugs, while those with $K C N Q 2$ encephalopathy reportedly have a positive response to sodium-channel blockers ${ }^{3,7,8)}$. In other words, the early identification of $K C N Q 2$ encephalopathy makes it a more direct therapeutic approach possible. Recently, KCNQ2 encephalopathy has been reported to have a characteristic amplitude-integrated electroencephalographic (aEEG) pattern ${ }^{9)}$. If there are characteristic findings on the aEEG, this distinct ictal pattern is also expected to be present on conventional electroencephalography (EEG). Early recognition of the electroclinical phenotype with aEEG monitoring can direct genetic testing and a precise therapeutic approach using sodium-channel blockers.

This report describes a case of KCNQ2 encephalopathy with the characteristic ictal aEEG pattern, which was confirmed on conventional EEG.

\section{CASE REPORT}

A 3-day-old female infant presented with repetitive myoclonic jerking movements in both her legs that had begun on the day. She was born via spontaneous vaginal delivery at a local hospital at a gestational age of 38 and 4/7 weeks, with a birth weight of 3.5 $\mathrm{kg}$ and no perinatal complications. There was no family history of epilepsy or developmental delay. Her spontaneous activities were decreased, but there were no other abnormalities (such as abnormal morphologic features) on physical examination. The results of her laboratory investigations, including metabolic profiles such as serum amino acids and urine organic acids, workup for infections and cerebrospinal fluid study were within normal ranges. Brain magnetic resonance imaging only revealed a low-grade cystic germinal matrix hemorrhage at the left caudothalamic groove (Figure 1).

In the neonatal intensive care unit (NICU), seizure monitoring was done using a continuous two-channel aEEG. The seizures lasted for a few minutes and were characterized by tonic posturing followed by unilateral or bilateral clonic jerking. They were always accompanied by apnea and desaturation, and sometimes preceded by crying. Ictal aEEG showed a sudden rise of the highest and lowest margins followed by burst suppression (Figure 2). Conventional EEG showed very frequent episodes of spike or sharp wave discharges, occasionally with a burst sup pression pattern. Ictal EEG also showed that the fast activities began from the right frontal area (Figure 3). Multiple antiepileptic drugs (phenobarbital, levetiracetam, topiramate, and phenytoin)
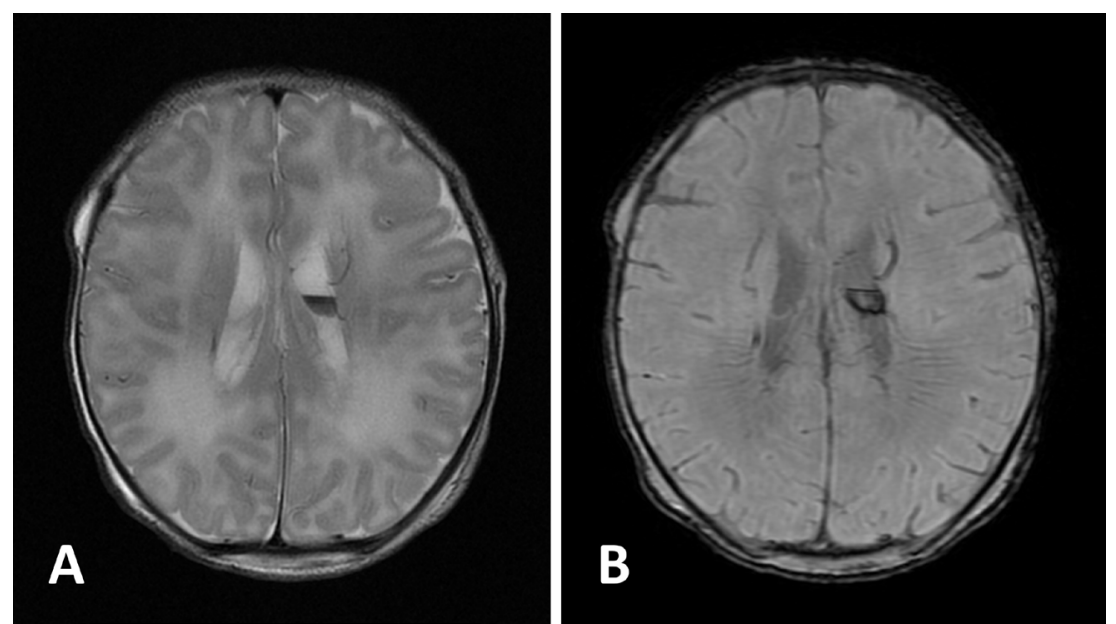

Figure 1. Brain magnetic resonance imaging of the patient with KCNQ2 encephalopathy. (A) T2-weighted imaging and (B) susceptibility-weighted imaging at day 2 of life showing cystic germinal matrix hemorrhage at the left caudothalamic groove. 
were administered; however, seizures and the characteristic ictal aEEG patterns were detected almost every day for over a month. The result of a chromosomal microarray, which was obtained approximately four weeks after testing, was normal. Genetic testing using next generation sequencing also was performed in the 6th week of life and a KCNQ2 mutation (c.794C>T; p.[Ala265Val]) was detected (Figure 4). Consequently, she was diagnosed with KCNQ2 encephalopathy.

At the age of 5 months, the patient continued to be seizurefree, and was being administered levetiracetam, topiramate,
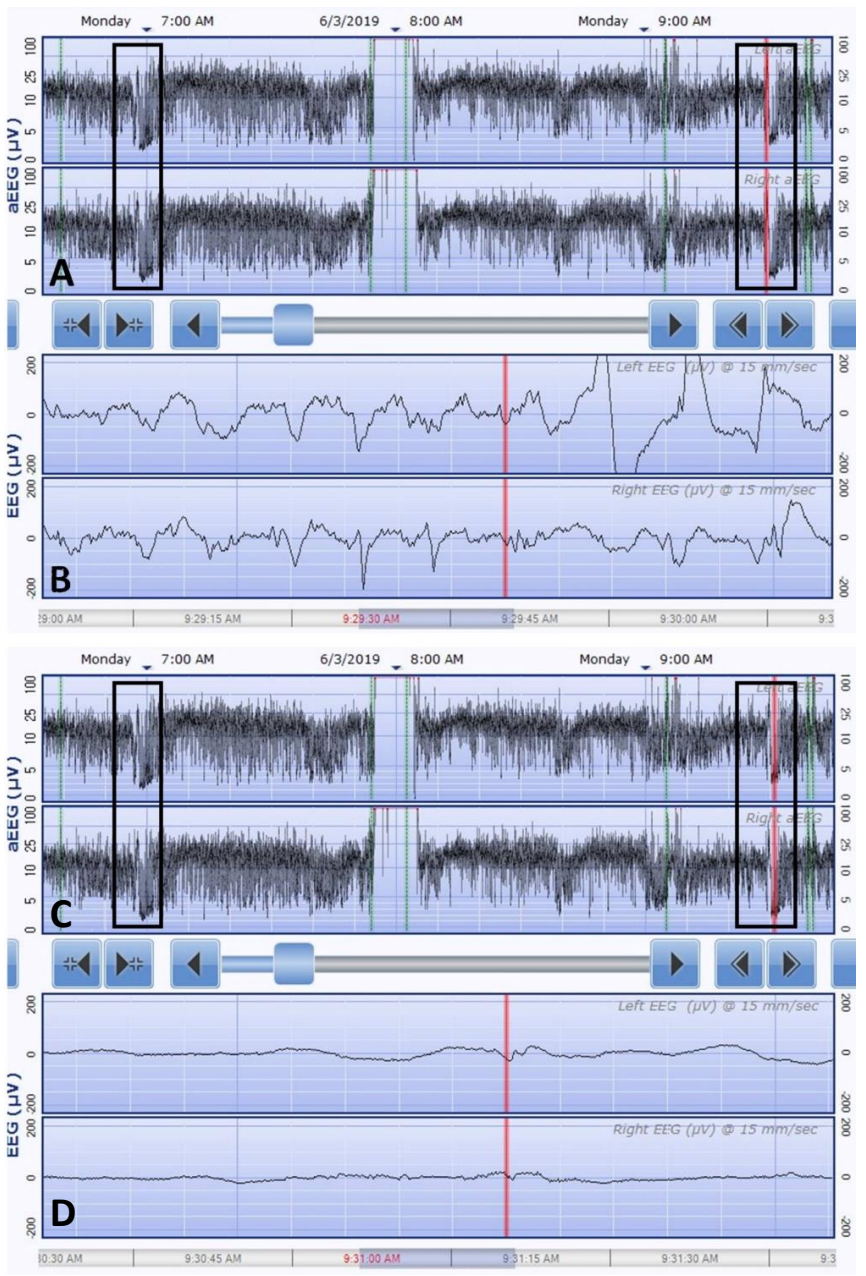

Figure 2. Seizure pattern of the patient with KCNQ2 encephalopathy on amplitude-integrated electroencephalography (aEEG). The upper panel displays the aEEG and the lower panel displays the corresponding electroencephalography (EEG) at that point indicated by the vertical red line. The aEEG shows two ictal discharges (box), a brief rise in amplitude in the upper and lower margins followed by suppression of the background pattern. The initial phase of the ictal discharges (red line in A) shows runs of spike and wave discharges on raw EEG (B). The later phase of the ictal discharges (red line in $\mathrm{C}$ ) shows diffuse background suppression on raw EEG (D). lamotrigine, and phenobarbital. She has been undergoing reha bilitation therapy and regular follow-up with developmental tests. A recent Denver Developmental Screening Test revealed a global developmental delay, and the specific delayed age in dif ferent domains noted at 12 months of age were: gross motor (7 months of age), fine motor ( 8 months of age), language ( 7 months of age), and psychosocial domains (8 months of age).

\section{DISCUSSION}

KCNQ2 encephalopathy is associated with a severe form of epilepsy characterized by recurrent tonic seizures with ictal apnea and desaturation, often followed by clonic movements, in the first week of life ${ }^{7,9,10}$. The seizures are extremely resistant to antiepileptic therapy resulting in severe developmental delay, regression, and various comorbidities.

In previous studies, sodium-channel blockers, such as carbamazepine and phenytoin, were reported to be effective in patients with KCNQ2 encephalopathy ${ }^{3,7,8,11)}$. Phenobarbital is the most commonly used antiepileptic drug for neonatal seizures, while carbamazepine is rarely used in neonates. Thus, an early diagnosis of $K C N Q 2$ encephalopathy allows more precise management, resulting in more favorable seizure and developmental outcomes. Identifying the etiology of neonatal seizures is crucial for diagnosing and managing seizures. Voltage-gated sodium channels and KCNQ2 potassium channels co-localize and modulate each other at the neuronal complex comprising the channels ${ }^{12,13)}$. This structure-function may explain the effective ness of sodium-channel blockers in patients with KCNQ2 encephalopathy ${ }^{13)}$.

Recently, a distinctive ictal pattern was reported during aEEG monitoring in infants with $K C N Q 2$ encephalopathy ${ }^{9}$. Continuous aEEG monitoring is widely used for the diagnosis of seizures in real time in the NICU ${ }^{14}$. Our patient also exhibited repeated seizures with the characteristic aEEG findings that have been described by Vilan et al. ${ }^{9)}$. They described that the aEEG patterns consist of an abrupt and short rise of the lower and upper margins followed by sudden depression of the background activities. They highlighted the importance of a distinctive aEEG pattern recognition for the genetic diagnosis and targeted treatment of this disorder. Our patient also demonstrated this distinctive aEEG pattern on brain monitoring when she experienced tonic or clonic seizures with apnea or desaturation. Moreover, her seizures 

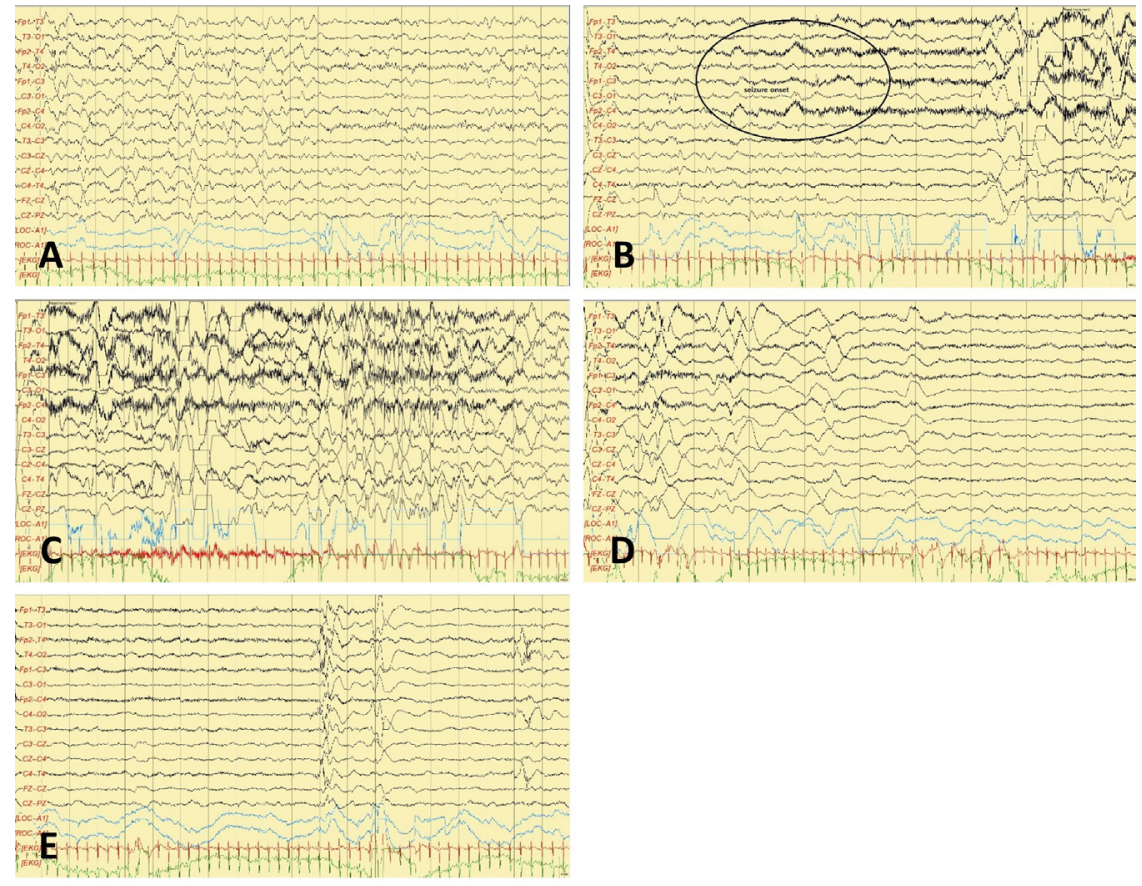

Figure 3. Seizure pattern of the patient with KCNQ2 encephalopathy on electroencephalography (EEG). (A) Interictal EEG, (B) ictal EEG shows that the fast activities began mainly from the right frontal area (circle, seizure onset), evolving to generalized spikes and waves (C) that were followed by generalized diffuse background attenuation. (D, E) Postictal EEG showing a burst suppression pattern.

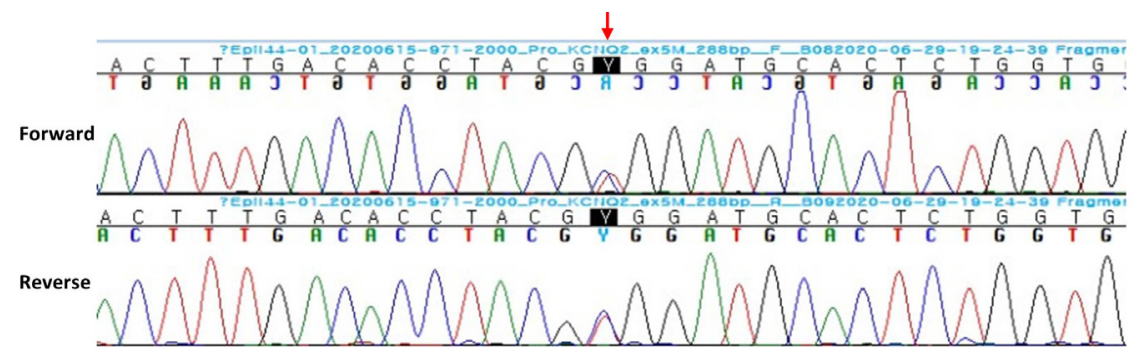

Figure 4. Sanger sequencing confirms KCNQ2 mutation [c.794C>T; p. (Ala265Val)] (arrow).

were vague, and she mainly experienced desaturation and apnea. She had persistent seizures despite the administration of multiple antiepileptic drugs, indicating epileptic encephalopathy; therefore, we conducted gene panel testing. Identifying this characteristic aEEG pattern of KCNQ2 encephalopathy can lead to early diagnosis and a trial of sodium-channel blockers such as carbamazepine in this condition. Thus, it could influence the selection of an appropriate antiepileptic drug.

This ictal pattern was also confirmed on conventional EEG (Figure 3). Episodes of apnea and desaturation are often underrecognized or misinterpreted as seizures by medical professionals. Therefore, the recognition of this characteristic aEEG or EEG pattern by medical professionals can increase their like- lihood of detecting seizures. Early recognition of this electro clinical phenotype by a neonatologist (aEEG) or a neurologist (EEG) can result in genetic testing, leading to the early diagnosis and effective treatment of $K C N Q 2$ encephalopathy with carba mazepine.

Identifying the characteristic ictal pattern on aEEG monitoring facilitates the early detection of KCNQ2 encephalopathy and timely treatment of seizures in patients with refractory seizures. Performing a targeted gene panel after recognizing the characteristic electroclinical phenotype may result in earlier diagnosis and more specific therapeutic management, thus contributing to an improved disease prognosis. 


\section{ARTICLE INFORMATION}

\section{Ethical statement}

This case report was reviewed and approved by the Institutional Review Board of Kyungpook National University Hospital (Approval number: 2020-04-013-002). As this was a retrospective case report, the requirement for written informed consent was waived.

\section{Conflicts of interest}

No potential conflict of interest relevant to this article was reported.

\section{Author contributions}

Conception or design: E.J.L.

Acquisition, analysis, or interpretation of data: N.K., Y.J.L., D.K., S.K.H., S.K., E.J.L.

Drafting the work or revising: N.K., Y.J.L.

Final approval of the manuscript: E.J.L.

\section{ORCID}

Naeun Kwakhttps://orcid.org/0000-0002-1526-4485

Eun Joo Lee https://orcid.org/0000-0003-1014-5783

\section{Acknowledgments}

None

\section{REFERENCES}

1. Scheffer IE, Berkovic S, Capovilla G, Connolly MB, French J, Guilhoto L, et al. ILAE classification of the epilepsies: position paper of the ILAE Commission for Classification and Terminology. Epilepsia 2017;58:512-21.

2. Reif PS, Tsai MH, Helbig I, Rosenow F, Klein KM. Precision medicine in genetic epilepsies: break of dawn? Expert Rev Neurother 2017;17:381-92.

3. Na JH, Shin S, Yang D, Kim B, Kim HD, Kim S, et al. Targeted gene panel sequencing in early infantile onset developmental and epileptic encephalopathy. Brain Dev 2020;42:438-48.

4. Milh M, Boutry-Kryza N, Sutera-Sardo J, Mignot C, Auvin S, Lacoste C, et al. Similar early characteristics but variable neurological outcome of patients with a de novo mutation of KCNQ2. Orphanet J Rare Dis 2013;8:80.

5. Biervert C, Schroeder BC, Kubisch C, Berkovic SF, Propping $\mathrm{P}$, Jentsch TJ, et al. A potassium channel mutation in neonatal human epilepsy. Science 1998;279:403-6.

6. Dedek K, Fusco L, Teloy N, Steinlein OK. Neonatal convulsions and epileptic encephalopathy in an Italian family with a missense mutation in the fifth transmembrane region of KCNQ2. Epilepsy Res 2003;54:21-7.

7. Numis AL, Angriman M, Sullivan JE, Lewis AJ, Striano $\mathrm{P}$, Nabbout R, et al. KCNQ2 encephalopathy: delineation of the electroclinical phenotype and treatment response. Neurology 2014;82:368-70.

8. Kuersten M, Tacke M, Gerstl L, Hoelz H, Stulpnagel CV, Borggraefe I. Antiepileptic therapy approaches in KCNQ2 relat ed epilepsy: a systematic review. Eur J Med Genet 2020;63: 103628.

9. Vilan A, Mendes Ribeiro J, Striano P, Weckhuysen S, Weeke LC, Brilstra E, et al. A distinctive ictal amplitude-integrated electroencephalography pattern in newborns with neonatal epilepsy associated with KCNQ2 mutations. Neonatology 2017; 112:387-93.

10. Sands TT, Balestri M, Bellini G, Mulkey SB, Danhaive O, Bakken $\mathrm{EH}$, et al. Rapid and safe response to low-dose carbamazepine in neonatal epilepsy. Epilepsia 2016;57:2019-30.

11. Shbarou R, Mikati MA. The expanding clinical spectrum of genetic pediatric epileptic encephalopathies. Semin Pediatr Neurol 2016;23:134-42.

12. Ambrosio AF, Silva AP, Malva JO, Soares-da-Silva P, Carvalho AP, Carvalho CM. Carbamazepine inhibits L-type Ca2+ channels in cultured rat hippocampal neurons stimulated with glutamate receptor agonists. Neuropharmacology 1999;38:1349-59.

13. Nguyen HM, Miyazaki H, Hoshi N, Smith BJ, Nukina N, Goldin $\mathrm{AL}$, et al. Modulation of voltage-gated $\mathrm{K}+$ channels by the sodium channel $\beta 1$ subunit. Proc Natl Acad Sci U S A 2012;109: 18577-82.

14. de Vries LS, Hellstrom-Westas L. Role of cerebral function monitoring in the newborn. Arch Dis Child Fetal Neonatal Ed 2005;90:F201-7. 